\title{
Should chloroquine and hydroxychloroquine be used to treat COVID-19? A rapid review
}

\section{Kome Gbinigie, MB BChir, MRCGP, DRCOG, DfSRH, PGCert (Health Research) ${ }^{1 \star \dagger}$, Kerstin Frie, BSc, MSc, DPhil (Oxon) ${ }^{2 \dagger}$}

${ }^{1} \mathrm{GP}$ and Doctoral Researcher, Nuffield Department of Primary Care Health Sciences, University of Oxford, Oxford, UK; ${ }^{2}$ Post-Doctoral Researcher, Nuffield Department of Primary Care Health Sciences, University of Oxford, Oxford, UK
*For correspondence:

Oghenekome.gbinigie@phc.ox. ac.uk

†These authors also contributed equally to this work

Competing interest: See page 6

Received: 29 March 2020

Accepted: 01 April 2020

Published: 08 April 2020

(C)This article is Open Access: CC BY license (https://creativecommons.org/licenses/by/4.0/)

Author Keywords: COVID-19, coronavirus, Chloroquine, Hydroxychloroquine, primary healthcare, general practice

Copyright (C) 2020, The Authors;

DOI:10.3399/

bjgpopen20X101069

\begin{abstract}
Background: On the 11 March 2020, the World Health Organization (WHO) declared that COVID-19 was a pandemic. To date, there are no medical treatments for COVID-19 with proven effectiveness. Novel treatments and/or vaccines will take time to be developed and distributed to patients. In light of this, there has been growing interest in the use of existing medications, such as chloroquine (CQ) and hydroxychloroquine ( $\mathrm{HCQ}$ ), as potential treatments of this disease.

Aim: To establish the current evidence for the effectiveness of $\mathrm{CQ}$ and $\mathrm{HCO}$ in treating COVID-19.

Design \& setting: A rapid review of the literature was conducted.

Method: Electronic searches in PubMed and Google Scholar were conducted on 21 March 2020. A further search was conducted in Google for relevant literature on 28 March 2020.

Results: There is limited evidence of in vitro activity of CQ/HCQ against SARS-CoV-2. A number of in vivo clinical trials are underway. The empirical data available from two of these trials reveal conflicting results. Both trials are characterised by small numbers of participants $(n=30$ and $n=36)$ and suffer methodological limitations. No medium or long-term follow-up data is available.

Conclusion: At present, there is insufficient evidence to determine whether $\mathrm{CQ} / \mathrm{HCO}$ are safe and effective treatments for COVID-19. High quality, adequately powered randomised clinical trials in primary and secondary care settings are urgently required to guide policymakers and clinicians. These studies should report medium- and long-term follow-up results, and safety data.
\end{abstract}

\section{How this fits in}

Chloroquine ( $\mathrm{CQ}$ ) and hydroxychloroquine ( $\mathrm{HCQ}$ ) have been used in the treatment and prophylaxis of a number of conditions, such as malaria, for several years. As novel treatments for COVID-19 are likely to take time to develop, a number of clinical trials have been registered to investigate the effectiveness of existing medications such as $\mathrm{CQ}$ or $\mathrm{HCQ}$. At present, there is insufficient evidence to recommend their use for the current pandemic outside of clinical trials. Further, high quality studies are urgently needed to provide timely guidance for clinicians and policymakers alike.

\section{Introduction}

To date, there have been over 17000 cases and 1000 deaths due to COVID-19 in the UK alone. ${ }^{1}$ There are intensive efforts worldwide to develop novel treatments for COVID-19 as the pandemic continues to spread. Such treatments will take time to develop and become available for widespread use. In 
the interim, there has been increasing interest in the use of existing medications to treat COVID-19, including $\mathrm{CQ}$ and $\mathrm{HCQ}$. $\mathrm{CQ}$ was first used as prophylaxis and treatment for malaria. $\mathrm{HCQ}$ is a more soluble and less toxic metabolite of $\mathrm{CQ}$. It causes fewer side effects and is therefore considered safer than CQ. ${ }^{2-4}$

The antiviral properties of both medications have been researched in recent years. $\mathrm{CQ}$ and $\mathrm{HCQ}$ have been used in the treatment of HIV with mixed results. ${ }^{5}$ The ability of these drugs to inhibit other coronaviruses, such as SARS-CoV-1, has been explored with promising results. ${ }^{6,7}$ There are a number of theories to explain the potential mechanism of action of CQ/HCQ against SARS-CoV-2. The virus is believed to upregulate cell surface angiotensin converting enzyme 2 (ACE2), ${ }^{8}$ binding to this enzyme in order to facilitate host cell entry. ${ }^{9} \mathrm{CQ}$ may reduce glycosylation of ACE2, thus preventing this attachment process. ${ }^{10}$ Another potential mechanism involves the inhibition of viral release into the intracellular space. Within the host cell, the virus is surrounded by a cell-membrane derived vesicle - the endosome - within which the virus replicates. ${ }^{11} \mathrm{CQ}$ is believed to accumulate in lysosomes, which may interrupt the usual process of lysosome-endosome fusion, thereby inhibiting release of the viral contents. ${ }^{11,12}$ Moreover, CQ may also block the production of interleukin- 6 and other proinflammatory cytokines, which are key mediators of acute respiratory distress syndrome (ARDS). ${ }^{11}$

Both drugs are affordable, widely available internationally, and are generally considered safe for their present US Food and Drug (FDA)-approved indications. However, these drugs have been incorporated into some national guidelines to treat COVID-19 in certain situations, despite a lack of rigorous clinical trial evidence of effectiveness. ${ }^{13,14}$ Furthermore, while generally considered safe, there are potential risks associated with taking these medications. Across the world there have been several reports of overdoses in people self-medicating with $\mathrm{CQ}$ during the current pandemic. ${ }^{15,16}$ There is therefore an urgent need to determine the safety and efficacy of these drugs for treating COVID-19, in order to guide international efforts to contain the pandemic. The aim of this rapid review is therefore to provide a timely summary of the evidence to date for the safety and effectiveness of $\mathrm{CQ} / \mathrm{HCO}$ as COVID-19 treatments.

\section{Method}

Electronic searches were conducted in PubMed and Google Scholar on 21 March 2020 using the search terms *chloroquine, coronavirus, SARS-Cov-2, 2019-NCov, and COVID-19. An additional search of Google was conducted on 28 March 2020 to find further relevant internet proceedings. Titles and abstracts of the search results were screened, and in vitro and in vivo studies assessing the use of CQ/ $\mathrm{HCQ}$ for the treatment of SARS-CoV-2 were included. In addition, reviews of the existing literature on this topic were included. No time, language, or study type restrictions were applied. A Chinese native language speaker helped to translate Chinese articles. Inclusion criteria were deliberately broad, to maximise the capture of potentially relevant studies, including pre-prints. Exclusion criteria were not applied. This article provides narrative summary of the current evidence.

\section{Results}

As described elsewhere, a limited number in vitro and in vivo studies have evaluated the use of CQ/ HCQ in treating SARS-CoV-2 infection. ${ }^{17}$

\section{In vitro studies}

There is some in vitro data supporting the ability of CQ and HCQ to inhibit SARS-CoV-2 activity. ${ }^{2,18,19}$ Wang et al found that $\mathrm{CQ}$ was highly selective in its activity against the virus rather than host cells. ${ }^{19}$ Liu et al $^{18}$ found a similar $50 \%$ cytotoxic concentration (CC50 - the concentration which results in $50 \%$ cell death) for $\mathrm{CQ}$ and $\mathrm{HCQ}$, and found that $\mathrm{CQ}$ was more potent than $\mathrm{HCQ}$ against the virus. By contrast, Yao et $\mathrm{al}^{2}$ found that HCQ was more potent against SARS-CoV-2.

\section{In vivo clinical trials}

Very limited in vivo data assessing the effectiveness of CQ/HCQ for COVID-19 has been published to date (see Table 1). The first reports of clinical effectiveness of CQ for COVID-19 came from a news briefing by the Chinese government in February 2020. They reported a positive signal from the results 


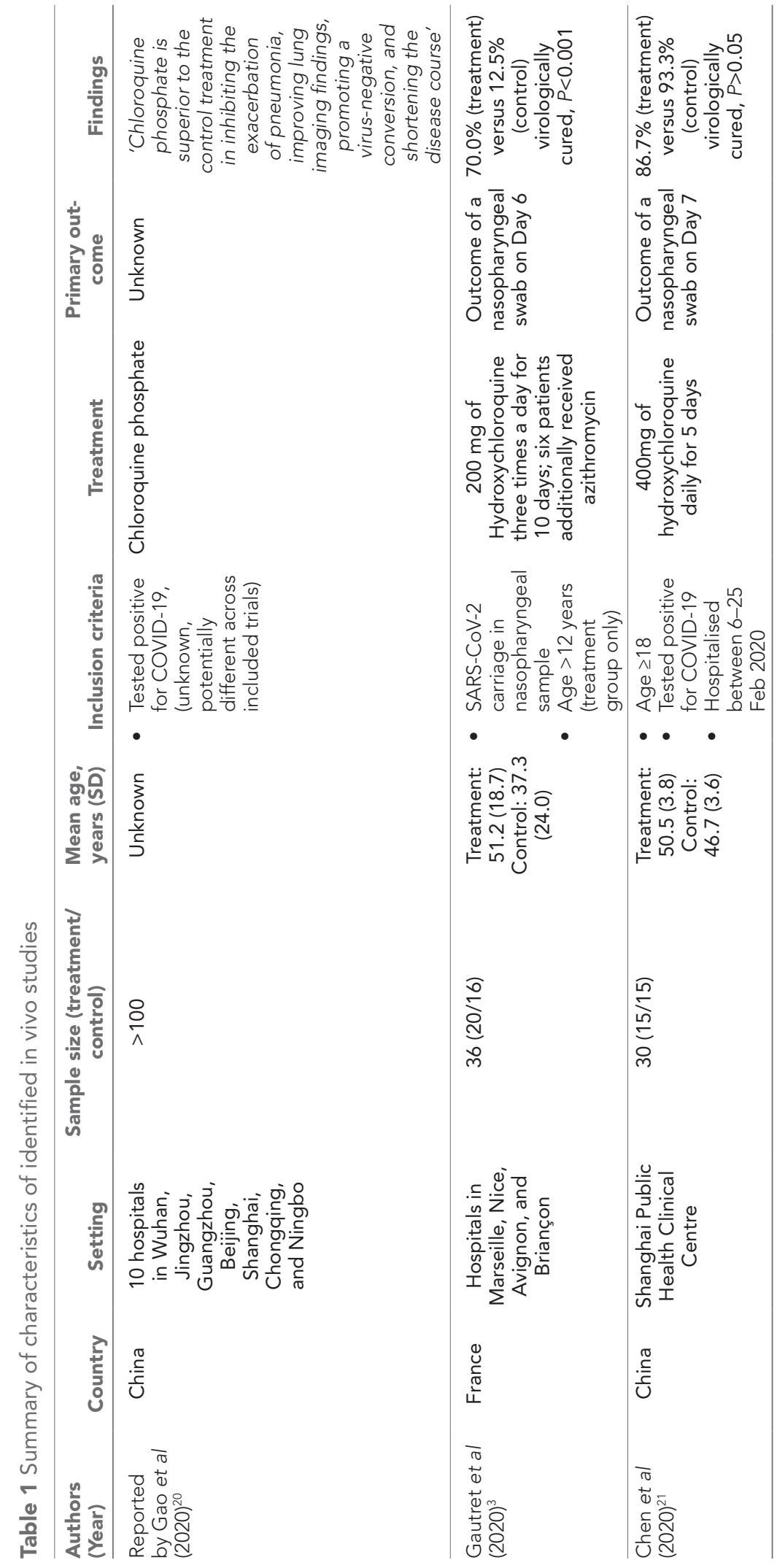


of treating more than 100 patients with $C Q$ in China, reportedly reducing the duration of illness, and improving COVID pneumonia and appearances on chest imaging. ${ }^{20}$ No adverse events were reported. These findings seem to be the combination of results from a number of ongoing clinical trials in different sites in China, using a variety of study protocols. No empirical data supporting these findings have been published thus far, and the evidence has therefore not been peer reviewed.

The first empirical data from a clinical trial was published on the 6 March 2020 by Chen and colleagues from Shanghai, China. ${ }^{21}$ They conducted a randomised controlled trial to test the effectiveness of $\mathrm{HCQ}$ in 30 adult patients who tested positive for COVID-19. Patients in the treatment group received $400 \mathrm{mg} \mathrm{HCQ}$ for 5 days, while the control group received usual care. The result of a nasopharyngeal swab on Day 7 was used as the primary outcome. The authors report three adverse events in the control group (one patient with abnormal liver function and anaemia, and one patient with abnormal renal function), and four adverse events in the treatment group (two patients with diarrhoea, one with lethargy, and one patient with abnormal liver function tests), which they argue were not linked to treatment with $\mathrm{HCO}$. One patient in the treatment group deteriorated significantly and thus $\mathrm{HCO}$ was stopped on Day 4 of the treatment. The intention-to-treat analysis revealed that the treatment group did not differ from the control group in the number of patients testing negative for COVID-19 on Day 7 (13 versus 14 patients), nor the duration of illness (all $P>0.05$ ).

The second article reporting clinical trial data came from a French study published by Gautret and colleagues on 17 March 2020. ${ }^{3}$ The researchers conducted an open-label, non-randomised controlled trial with 36 patients diagnosed with COVID-19, of which six were asymptomatic, 22 had upper respiratory tract infection symptoms, and eight had lower respiratory tract infection symptoms. The twenty patients in the treatment group received $\mathrm{HCO} 200 \mathrm{mg}$ three times a day for 10 days. Patients declining to take part in the study and not meeting the inclusion criteria were assigned to the control group and received usual care. Six of the patients in the treatment group additionally received azithromycin to prevent bacterial superinfection. The primary outcome was SARS-CoV-2 carriage at Day 6, detected from polymerase chain reaction (PCR) of SARS-CoV-2 ribonucleic acid (RNA) on nasopharyngeal swabs.

Patients in the treatment group were significantly more likely to test negative for SARS-CoV-2 on Day 6 than controls $(70 \%$ versus $12.5 \%$ virologically cured, $P<0.001$ ). All six patients treated with $\mathrm{HCO}$ and azithromycin tested negative on Day 6 . The authors argue that these findings provide preliminary evidence of effectiveness of $\mathrm{HCQ}$, and possible synergy between $\mathrm{HCQ}$ and azithromycin.

\section{Limitations of the current empirical data}

The in vivo studies reported have methodological deficiencies. The trials by Chen et al and Gautret and colleagues were both underpowered according to their own calculations, which may have led to an exaggeration of effect sizes and false positive results. ${ }^{22}$ Moreover, whilst viral status at Day 6/ Day 7 were the primary outcomes, no medium- or long-term follow-up data were presented in either study. Gautret and colleagues reported that one patient tested negative for the virus on Day 6, but subsequently tested positive on Day 8. This highlights the need for long-term follow-up data. Chen et al reported that most patients included in their trial (both control and treatment group) recovered quickly from COVID-19, indicating that the researchers had recruited a sample with mild symptoms. The authors argue that the lack of severe cases may have caused a ceiling effect, which may explain the non-significant results. Gautret et a $\left.\right|^{3}$ did not perform intention-to-treat analysis, excluding six patients from their analyses who had dropped out at follow-up, which may have introduced bias. ${ }^{23}$ Finally, Gautret and colleagues did not randomise patients to the control and treatment group, potentially introducing allocation bias.

\section{Discussion}

\section{Summary}

There is limited evidence suggesting in vitro activity of CQ/HCQ against SARS-CoV-2. The available in vivo empirical data is limited to two studies, with very small sample sizes, a number of methodological flaws, and conflicting results. On the basis of preliminary results from ongoing clinical trials, some countries have incorporated $\mathrm{CQ} / \mathrm{HCO}$ into their treatment protocols for certain patients with COVID-19. There is presently no medium to long-term follow-up data to support this approach. The 
very limited safety data available has not revealed serious side effects of these medications in the context of treating COVID-19.

\section{Strengths and limitations}

To the authors' knowledge, this is the first review that reports empirical data of the effectiveness of CQ/HCQ for treating COVID-19. By collaborating with a Chinese native language speaker, the authors were able to incorporate not only English articles, but also a Chinese report. This review was conducted quickly (10 days from searches being conducted to journal submission) in order to facilitate timely dissemination of the findings. As a result, however, these searches were not as rigorous as those of a systematic review. Some potentially relevant studies may therefore have been missed, particularly unpublished studies. The authors deliberately kept their search strategy broad to try to mitigate this and did not apply any exclusion criteria. Given the rapidly increasing number of patients infected with SARS-CoV-2 and the associated morbidity and mortality, the authors believe that the methodology employed was appropriate in this context.

\section{Comparison with existing literature}

Two systematic reviews that have been conducted in this area were identified. ${ }^{24,25}$ Cortegiani et al ${ }^{24}$ identified one in vitro study, ${ }^{19}$ a narrative letter reporting preliminary findings of several ongoing clinical trials in China, ${ }^{20}$ an editorial, ${ }^{26}$ an expert consensus paper, ${ }^{27}$ and two national guideline documents (Dutch and Italian). The review also provides an overview of the methodology of 23 ongoing clinical trials in China. The authors conclude that there are grounds to conduct high quality trials of CQ/HCQ for COVID-19. They did not report any empirical data for clinical trials of CQ/HCQ. A systematic review by Singh and colleagues ${ }^{25}$ identified two articles reporting the results of in vivo studies, which are also captured in this rapid review. ${ }^{3,20}$ Singh et al excluded studies written in Chinese, and therefore did not report the findings of the Chinese pilot study by Chen and colleagues. ${ }^{21}$ The present rapid review therefore serves as an important update, and reports more empirical data from in vivo studies than any other review on this topic, to the best of the authors' knowledge.

There is limited safety data available for the use of CQ/HCQ in the context of COVID-19. In the report of over 100 patients in ongoing Chinese clinical trials presented in a news briefing by the Chinese government, ${ }^{20}$ no adverse events were reported. In the pilot study by Chen et al, ${ }^{21}$ adverse events related to gastrointestinal disturbance were only reported for the treatment group. However, the sample size makes it difficult to judge whether these side effects were caused by treatment with HCQ. Gautret and colleagues reported no adverse events, but stated that data on side effects would be published in a separate paper. $\mathrm{CQ} / \mathrm{HCQ}$ are generally considered safe medications for their present FDAapproved indications; ${ }^{10}$ however, their prescription is not without risk. Gastrointestinal upset has been reported with HCQ. ${ }^{28}$ Retinal toxicity has been described with long-term use, ${ }^{29,30}$ and overdosage $^{30,31}$ of $\mathrm{CQ} / \mathrm{HCQ}$. There have been isolated reports of cardiomyopathy ${ }^{32}$ and arrhythmia ${ }^{33}$ caused by treatment with $\mathrm{CQ}$. $\mathrm{CQ}$ should be avoided in patients with porphyria. ${ }^{34}$ Both $\mathrm{CQ}$ and $\mathrm{HCQ}$ are metabolised in the liver with renal excretion of some metabolites, hence renal and liver function must be taken into account when these medications are prescribed. ${ }^{34,35}$ In a letter to the editor, Risambaf et a/ ${ }^{34}$ raises concern about COVID-19 inducing liver and renal impairment, which may increase the risk of toxicity of CQ/HCQ when used to treat COVID-19. Patients' comorbidities should be carefully considered before these drugs are prescribed.

\section{Implications for research and practice}

Given the considerable limitations of the current available evidence, there is an urgent need for more, adequately powered, high quality randomised clinical trials to enable a better understanding of the effectiveness of CQ and HCQ for treating COVID-19. These studies should rigorously report safety data, as well as medium- and long-term follow-up data once this becomes available. Fortunately, more than 20 clinical trials are already registered to achieve this goal. ${ }^{36}$ These trials should help to inform the optimal dose and duration of treatment. All of the studies identified in this rapid review were conducted in secondary care. Studies in primary care settings are also needed to evaluate whether $\mathrm{CQ} / \mathrm{HCQ}$ has utility in community settings. The authors are aware of one trial that has been registered to this purpose. ${ }^{37}$ 
The results of these studies should be made available in a timely fashion and to an international audience. This will facilitate synthesis of the results and enable important decisions to be made about the utility and safety of these drugs for the current COVID-19 pandemic. HCQ has a better safety profile, and may therefore be the preferred drug to focus initial research efforts on.

At present, there is insufficient evidence to recommend the prescription of these drugs by primary or secondary care physicians for COVID-19 outside of the context of research.

In conclusion, a limited number of in vitro studies report antiviral activity of $\mathrm{CQ}$ and $\mathrm{HCQ}$ against SARS-CoV-2. The data from in vivo studies is very limited, with mixed findings, which are likely to be prone to bias due to methodological limitations. Further research is now urgently needed in both primary and secondary care to determine whether $\mathrm{CQ} / \mathrm{HCQ}$ are safe and effective treatments for COVID-19. At present, there is insufficient evidence to support their use in the management of COVID-19 outside of research.

\section{Funding}

OAG's time on the review was funded by the Wellcome Trust (Grant reference code 203921/Z/16/Z). KF's time on the review was funded by the Wellcome Trust, Our Planet Our Health (Livestock, Environment and People - LEAP), award number 205212/Z/16/Z. The views expressed are those of the authors and not necessarily those of the Wellcome Trust.

\section{Ethical approval}

N/A

\section{Provenance}

Freely submitted; externally peer reviewed.

\section{Acknowledgements}

OAG and KF are joint first authors of this article.

The authors would like to thank Christina Tang for her help in translating the paper published by Chen and colleagues.

\section{Competing interests}

$\mathrm{OAG}$ and $\mathrm{KF}$ are in receipt of funding from the Wellcome Trust.

\section{References}

1. Worldometer. Total Coronavirus Cases in the United Kingdom. 2020; https://www.worldometers.info/coronavirus/ country/uk/ (accessed 6 Apr 2020).

2. Yao $X, Y e F$, Zhang $M$, et al. In vitro antiviral activity and projection of optimized dosing design of hydroxychloroquine for the treatment of severe acute respiratory syndrome coronavirus 2 (SARS-CoV-2). Clin Infect Dis 202009 Mar 2020. DOI: https://doi.org/10.1093/cid/ciaa237

3. Gautret $\mathrm{P}$, Lagier J-C, Parola $\mathrm{P}$, et al. Hydroxychloroquine and azithromycin as a treatment of COVID-19: results of an open-label non-randomized clinical trial. Int J Antimicrob Agents 2020; 105949: 105949. DOI: https://doi.org/ 10.1016/j.ijantimicag.2020.105949

4. Sahraei Z, Shabani M, Shokouhi S, Saffaei A. Aminoquinolines against coronavirus disease 2019 (COVID-19): chloroquine or hydroxychloroquine. Int J Antimicrob Agents 2020; 105945.

5. Chauhan A, Tikoo A, AJHm T. The enigma of the clandestine association between chloroquine and HIV-1 infection. HIV Med 2015; 16(10): 585-590. DOI: https://doi.org/10.1111/hiv.12295

6. Keyaerts E, Li S, Vijgen L, et al. Antiviral activity of chloroquine against human coronavirus OC43 infection in newborn mice. Antimicrob Agents Chemother 2009; 53(8): 3416-3421. DOI: https://doi.org/10.1128/AAC.0150908

7. Vincent MJ, Bergeron E, Benjannet S, et al. Chloroquine is a potent inhibitor of SARS coronavirus infection and spread. Virol J 2005; 2(1): 69. DOI: https://doi.org/10.1186/1743-422X-2-69

8. Wang $\mathrm{P}-\mathrm{H}$, Cheng $\mathrm{Y}$. Increasing host cellular receptor-angiotensin-converting enzyme 2 (ACE2) expression by coronavirus may facilitate 2019-nCoV infection. [preprint]. bioR $\chi$ iv 2020.

9. Zhou $P$, Yang $X-L$, Wang $X-G$, et al. A pneumonia outbreak associated with a new coronavirus of probable bat origin. Nature 2020; 579(7798): 270-273. DOI: https://doi.org/10.1038/s41586-020-2012-7

10. Devaux CA, Rolain J-M, Colson P, Raoult D. New insights on the antiviral effects of chloroquine against coronavirus: what to expect for COVID-19? Int J Antimicrob Agents 2020: 105938. DOI: https://doi.org/10.1016/j. ijantimicag.2020.105938 
11. Savarino A, Boelaert JR, Cassone A, et al. Effects of chloroquine on viral infections: an old drug against today's diseases? Lancet Infect Dis 2003; 3(11): 722-727. DOI: https://doi.org/10.1016/S1473-3099(03)00806-5

12. Golden EB, Cho HY, Hofman FM, et al. Quinoline-based antimalarial drugs: a novel class of autophagy inhibitors. Neurosurg Focus 2015; 38(3): E12. DOI: https://doi.org/10.3171/2014.12.FOCUS14748

13. Dong L, Hu S, Gao J. Discovering drugs to treat coronavirus disease 2019 (COVID-19). Drug Discov Ther 2020; 14(1): 58-60. DOI: https://doi.org/10.5582/ddt.2020.01012

14. Nicastri E, Petrosillo N, Bartoli TA, et al. National Institute for the Infectious Diseases "L. Spallanzani", IRCCS Recommendations for COVID-19 clinical management. Infect Dis Rep 2020; 12(1): 8543. DOI: https://doi.org/10. 4081/idr.2020.8543

15. Busari S, Adebayo B. Nigeria records chloroquine poisoning after Trump endorses it for coronavirus treatment.CNN [online] 2020 Mar 23; https://edition.cnn.com/2020/03/23/africa/chloroquine-trump-nigeria-intl/ index.html (accessed 6 Apr 2020).

16. Beaumont $P$, Ratcliffe R. Chloroquine: Trump's misleading claims spark hoarding and overdoses. The Guardian [online] 2020 Mar 25; https://www.theguardian.com/science/2020/mar/25/can-chloroquine-really-help-treatcoronavirus-patients (accessed 6 Apr 2020).

17. Frie K, Gbinigie OA. Chloroquine and hydroxychloroquine: Current evidence of effectiveness in COVID-19. 2020; https://www.cebm.net/covid-19/chloroquine-and-hydroxychloroquine-current-evidence-for-their-effectiveness-intreating-covid-19/ (accessed 6 Apr 2020).

18. Liu J, Cao R, Xu M, et al. Hydroxychloroquine, a less toxic derivative of chloroquine, is effective in inhibiting SARSCoV-2 infection in vitro. Cell Discov 2020; 18(6): 16. DOI: https://doi.org/10.1038/s41421-020-0156-0

19. Wang $M$, Cao $R$, Zhang $L$, et al. Remdesivir and chloroquine effectively inhibit the recently emerged novel coronavirus (2019-nCoV) in vitro. Cell Res 2020; 30(3): 269-271. DOl: https://doi.org/10.1038/s41422-020-0282-0

20. Gao J, Tian Z, Yang X. Breakthrough: chloroquine phosphate has shown apparent efficacy in treatment of COVID-19 associated pneumonia in clinical studies. Biosci Trends 2020; 14(1): 72-. DOI: https://doi.org/10.5582/ bst.2020.01047

21. Chen J, Liu D, Liu L, et al. A pilot study of hydroxychloroquine in treatment of patients with common coronavirus disease-19 (COVID-19). Journal of ZheJiang University (Medical Sciences) 2020; 49(1).

22. Dumas-Mallet $\mathrm{E}$, Button KS, Boraud $\mathrm{T}$, et al. Low statistical power in biomedical science: a review of three human research domains. $R$ Soc Open Sci 2017; 4(2): 160254. DOI: https://doi.org/10.1098/rsos.160254

23. Ranganathan $P$, Pramesh CS, Aggarwal R. Common pitfalls in statistical analysis: intention-to-treat versus perprotocol analysis. Perspect Clin Res 2016; 7(3): 144-146. DOI: https://doi.org/10.4103/2229-3485.184823

24. Cortegiani A, Ingoglia $G$, Ippolito $M$, et al. A systematic review on the efficacy and safety of chloroquine for the treatment of COVID-19. J Crit Care 2020 DOI: https://doi.org/10.1016/j.jcrc.2020.03.005

25. Singh AK, Singh A, Shaikh A, et al. Chloroquine and hydroxychloroquine in the treatment of COVID-19 with or without diabetes: a systematic search and a narrative review with a special reference to India and other developing countries. Diabetes Metab Syndr 2020; 14(3): 241-246. DOI: https://doi.org/10.1016/j.dsx.2020.03.011

26. Colson P, Rolain J-M, Raoult D. Chloroquine for the 2019 novel coronavirus SARS-CoV-2. Int J Antimicrob Agents 2020; 55(3): 105923. DOI: https://doi.org/10.1016/j.ijantimicag.2020.105923

27. Jie Z, He H, Xi H, Zhi Z., Multicenter Collaboration Group of Department of Science and Technology of Guangdong Province and Health Commission of Guangdong Province for Chloroquine in the Treatment of Novel Coronavirus Pneumonia Expert consensus on chloroquine phosphate for the treatment of novel coronavirus pneumonia. Zhonghua Jie He He Hu Xi Za Zhi 2020; 43: E019. DOI: https://doi.org/10.3760/cma.j.issn.1001-0939.2020.0019

28. Srinivasa A, Tosounidou S, Gordon C. Increased incidence of gastrointestinal side effects in patients taking hydroxychloroquine: a brand-related issue? J Rheumatol 2017; 44(3): : 398. DOI: https://doi.org/10.3899/jrheum. 161063

29. Mavrikakis M, Papazoglou S, Sfikakis PP, et al. Retinal toxicity in long term hydroxychloroquine treatment. Ann Rheum Dis 1996; 55(3): 187-189. DOI: https://doi.org/10.1136/ard.55.3.187

30. Easterbrook M. The ocular safety of hydroxychloroquine. Semin Arthritis Rheum 1993; 23(2 Suppl 1): 62-. DOI: https://doi.org/10.1016/S0049-0172(10)80009-5

31. Browning DJ. Hydroxychloroquine and chloroquine retinopathy: screening for drug toxicity. Am J Ophthalmol 2002; 133(5): 649-656. DOI: https://doi.org/10.1016/s0002-9394(02)01392-2

32. Iglesias Cubero G, Rodriguez Reguero JJ, Rojo Ortega JM. Restrictive cardiomyopathy caused by chloroquine. $\mathrm{Br}$ Heart J 1993; 69(5): 451-452. DOI: https://doi.org/10.1136/hrt.69.5.451

33. Costedoat-Chalumeau N, Hulot JS, Amoura Z, et al. Heart conduction disorders related to antimalarials toxicity: an analysis of electrocardiograms in 85 patients treated with hydroxychloroquine for connective tissue diseases. Rheumatology (Oxford) 2007; 46(5): 808-810. DOI: https://doi.org/10.1093/rheumatology/kel402

34. Rismanbaf A, Zarei S. Liver and kidney injuries in COVID-19 and their effects on drug therapy; a letter to editor. Arch Acad Emerg Med 2020; 8(1).e17.

35. Wang Y, Zhu LQ. Pharmaceutical care recommendations for antiviral treatments in children with coronavirus disease 2019. World J Pediatr 202012 Mar 2020. DOI: https://doi.org/10.1007/s12519-020-00353-5

36. Aronson JK, Ferner RE, DeVito N, Heneghan C. COVID-19 trials registered up to 8 March 2020 - an analysis of 382 studies. The Centre for Evidence-Based Medicine. 2020; https://www.cebm.net/covid-19/registered-trials-andanalysis/ (accessed 6 Apr 2020).

37. Butler C, Ogburn E, Allen J, et al. Platform randomised trial of interventions against COVID-19 in older people (PRINCIPLE). [ISRCTN86534580]. ISRCTN 2020 